\title{
Evidence for cytochrome $f$ involvement in eggplant cell death induced by palmitoleic acid
}

\author{
Cell Death and Differentiation (2005) 12, 405-407. doi:10.1038/sj.cdd.4401551 \\ Published online 11 February 2005
}

\section{Dear Editor,}

In response to certain stimuli, animal and plant cells activate genetic and biochemical programs resulting in cell death, a process known as PCD. ${ }^{1}$ A large volume of information on animal PCD is now available. ${ }^{2,3}$ In contrast, less is known about plant PCD. It has been shown that there are certain morphological and biochemical similarities between plant and animal PCD. ${ }^{4}$ Thus, it is useful to compare the PCD of plants and animals in developing strategies to study PCD in plants.

One of the features found in certain PCD is the release of cyt $c$ from mitochondria to the cytosol. ${ }^{1,5,6}$ This initiates a process leading to DNA fragmentation, and eventually to biochemical execution of the cell. ${ }^{7}$ The evolutionary and biological significance of the involvement of the mitochondrion, an organelle known for its life-sustaining role with its energy conversion ability, in PCD is not clear. Plant cells have another energy conversion organelle, that is, the chloroplast. While the mitochondrion converts one form of chemical energy to another, the chloroplast converts light energy to chemical energy. In spite of this difference, both organelles utilize electron transport chains in their energy conversion. There are similarities as well as variations among the components of the electron transport chains of these two organelles. For example, cyt $f$ is found in chloroplasts but not in mitochondria. Cyt $f$ is a $c$-type cytochrome characterized by the covalent attachment of the heme to the polypeptide chain via thioether linkages, which are formed from two vinyl groups of heme and two cysteine residues provided by the $\mathrm{CXXCH}$ motif sequence ( $X$ denotes any amino acid). ${ }^{8}$

We have previously found that cyt $f$ release from eggplant chloroplasts could be induced by palmitoleic acid $(16: 1) .{ }^{9} \mathrm{We}$ have also observed that 16:1 induces death of eggplant cells. While the chloroplast has been found to be associated with certain forms of cell death ${ }^{10,11}$ we are not aware of any information on the involvement of cyt $f$ in the process. Here, we report our investigation on whether cyt $f$ release plays a role in the death of eggplant cells.

Cultured eggplant cells treated with 16:1 underwent cytoplasmic shrinkage and condensation (data not shown). 16:1 also caused shrinkage and collapse of eggplant protoplasts (Figure 1a). As it was easier to monitor protoplasts than irregularly shaped cells and cell colonies, we used protoplasts in this study. Death of protoplasts was accompanied by changes in their nuclei. Nuclei of untreated protoplasts stained with DAPI (4,6-diamidino-2-phenylindole) exhibited bright blue fluorescence which stayed unchanged throughout the experiment (Figure 1b), but the fluorescence of the nuclei of the treated protoplasts gradually became diffuse, dispersed, and eventually disappeared (Figure 1a). These changes were concurrent with protoplast shrinkage and collapse. DNA damage was also revealed by the TUNEL assay for nicked DNA and DNA breaks ${ }^{12}$ as 16:1 treatment rendered the protoplasts TUNEL positive (Figure 1c).

Release of cyt $f$ was probed with an antiserum raised against a synthetic peptide corresponding to the first 21 amino acids of the mature $\mathrm{N}$-terminus of the cyt $f$ protein. Two cyt $f$ proteins (38 and the $39 \mathrm{kDa}$ ) were found in the membrane fraction and none in the cytosolic fraction. 16:1 treatment led to the disappearance of the $38 \mathrm{kDa}$ cyt $f$ from the membranes and its appearance in the cytosol, suggesting its release from the membranes into the cytosol (Figure 1d). The release was detected within $10 \mathrm{~min}$ of treatment (Figure 1e). Thus, cyt $f$ release apparently preceded protoplast collapse and nuclear disintegration, which was not evident until $20 \mathrm{~min}$ after treatment.

16:1 could directly affect chloroplasts (Figure 1f). Intact chloroplasts possess three membranes (outer, inner, and thylakoid). Under a phase contrast microscope an isolated chloroplast is seen to possess a halo, which is dependent on the integrity of the outer and the inner envelopes. ${ }^{13}$ As such, the halo is a convenient marker for chloroplast integrity. 16:1 treatment was found to cause shrinkage of the outer membrane, but swelling of the area surrounding the thylakoid membranes, leading to the rupture of the chloroplast as manifested by the disappearance of its halo within approximately $7.5 \mathrm{~min}$ (Figure 1f). Electron microscopy study showed that the in vivo chloroplasts within a protoplast treated with 16:1 also collapsed but it took over 12 min (Figure 1g, h). The release of cyt $c$ from mitochondria has been found to be associated with the swelling and rupture of mitochondria. ${ }^{14}$ Chloroplast rupture was also associated with cyt $f$ release (Figure 1i). In untreated chloroplasts, cyt $f$ proteins ( 38 and $39 \mathrm{kDa}$ ) were only found in the thylakoid membranes (Figure 1i). Following 16:1 treatment, while the 39-kDa cyt fremained in the thylakoids, the 38-kDa cyt $f$ now was found in the stromal extract (Figure $1 \mathrm{j}$ ).

It has been demonstrated that $\mathrm{Ba}^{2+}$ is able to block both cyt $c$ release and death of Hela cells treated with apoptotic-inducing agents. ${ }^{15} \mathrm{Ba}^{2+}$ was also found to prevent the release of cyt $f$ and provide relatively effective protection to the protoplasts from collapse and nuclear damage induced by 16:1 (Figure 1k, 1I). Spermine (Spm) also prevented cyt $f$ release, but was less effective in protecting the protoplasts from collapse (Figure 1I, 

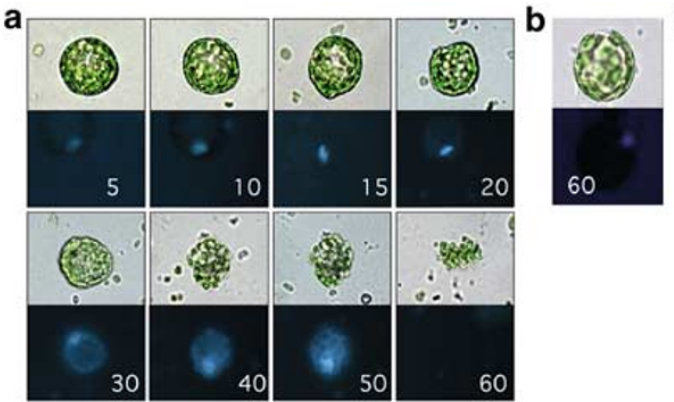

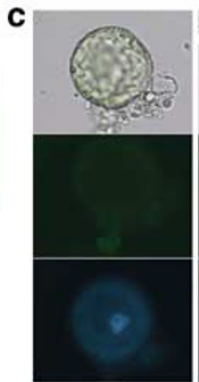

Control

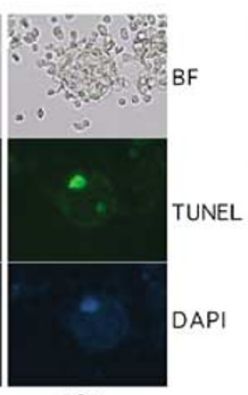

$16: 1$
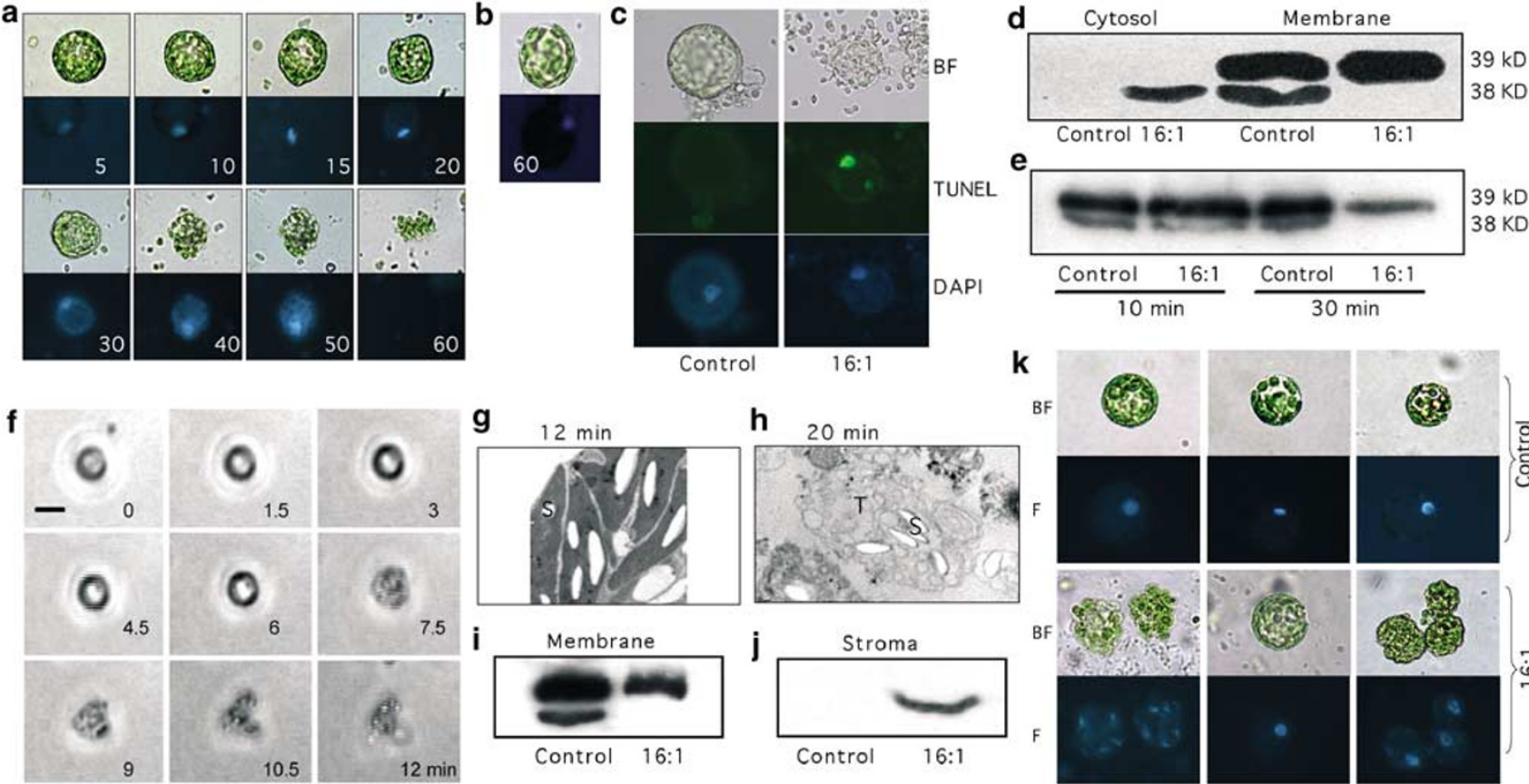

Control 16:1

h $20 \mathrm{~min}$
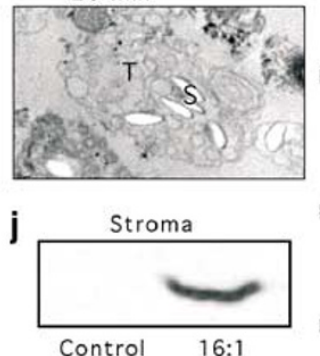

$\mathbf{k}$
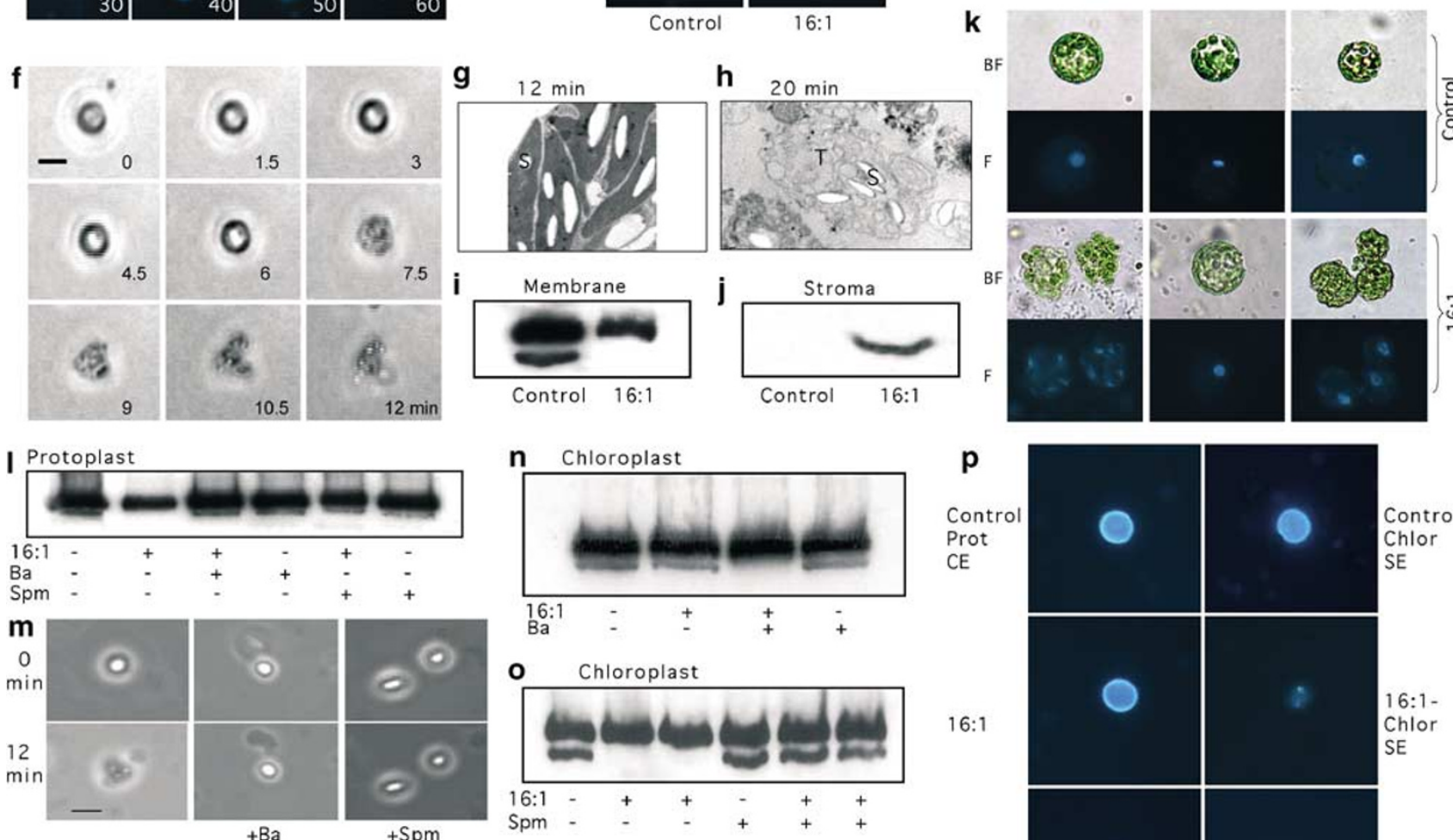

$16: 1$
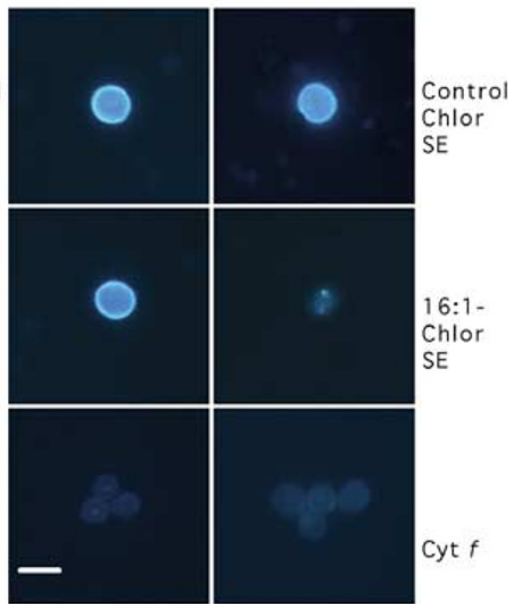

Figure 1 (a) Bright field (BF) and fluorescent (F) images of representative protoplasts treated with $400 \mu \mathrm{M}$ 16:1. Protoplasts were stained with DAPI. Unless otherwise mentioned, 16:1 was dissolved in 0.4\% DMSO, and 0.4\% DMSO was used as control in all experiments. (b) Representative control protoplast stained with DAPI. (c) Representative images of control (in 0.2\% DMSO) and treated protoplasts (with $200 \mu \mathrm{M} \mathrm{16:1} \mathrm{in} \mathrm{0.2 \%} \mathrm{DMSO)} \mathrm{labeled} \mathrm{with} \mathrm{TUNEL} \mathrm{and} \mathrm{counterstained} \mathrm{with} \mathrm{DAPI} \mathrm{and}$ viewed at BF, $450 / 490$ and $380 / 510 \mathrm{~nm}$. (d) Changes in cyt $f$ location following 16:1 treatment (400 $\mu \mathrm{M}$ for $45 \mathrm{~min}$ ) in protoplasts. Presence of cyt $f$ was assessed by immunoblotting using N-terminal-specific cyt $f$ antibody. (e) Time line of cyt $f$ release from thylakoid membranes of protoplasts treated with $400 \mu \mathrm{M} 16: 1$. (f) Phase contrast images of a representative chloroplast treated with $50 \mu \mathrm{M} \mathrm{16:1}$. The bar indicates $5 \mu \mathrm{m}$. (g, h) Transmission electron micrographs of in vivo chloroplasts within protoplasts treated with $50 \mu \mathrm{M}$ 16:1. The bar indicates $5 \mu \mathrm{m}$. T, thylakoid membrane; S, starch granule. (i, j) Distribution of cyt $f$ in isolated chloroplasts treated with $50 \mu \mathrm{M}$ 16:1 for $60 \mathrm{~min}$ and probed with cyt $f$. (k) BF and fluorescent $(380 / 510 \mathrm{~nm})$ microscopy of corresponding protoplasts stained with DAPI. Control (upper panels), 16:1 (lower panels). Treatments from left to right are: No addition, $+5 \mathrm{mM} \mathrm{Ba}^{2+}$, and $+1 \mathrm{~mm} \mathrm{Spm}$. Pictures were taken after $1 \mathrm{~h}$ of treatment. (I) Effects of $16: 1$, Spm, and $\mathrm{Ba}^{2+}$ on release of cyt $f$ from protoplasts probed with cyt $f$ antibody. $(\mathbf{m})$ Phase contrast images show $\mathrm{Ba}^{2+}$ and Spm-protected chloroplasts from 16:1 treatment $(50 \mu \mathrm{M})$. From left to right: control, $+5 \mathrm{mM} \mathrm{Ba}^{2+}$, and $+1 \mathrm{mM} \mathrm{Spm}$. The bar indicates $5 \mu \mathrm{m}$. (n, o) Release of cyt $f$ induced by $50 \mu \mathrm{M} 16: 1$ for $60 \mathrm{~min}$ from thylakoid membranes was prevented by $5 \mathrm{~mm} \mathrm{Ba}^{2+}$ or $1 \mathrm{~mm} \mathrm{Spm}$. (p) Fluorescent images $(380 / 485 \mathrm{~nm}$ ) of DAPI-stained isolated eggplant nuclei treated with 16:1, Cyt $f$, and cytosolic extracts (CE) from protoplasts and stromal extract (SE) from chloroplasts. All nuclei except for (c) were treated with cytosolic extract from control protoplasts. The images from top to bottom, right to left: CE from control protoplasts, $+50 \mu \mathrm{M}$ 16:1, CE from 16:1-treated protoplast, + SE from control chloroplasts, + SE from 16:1-treated chloroplasts, and + Cyt $f(2 \mu \mathrm{M})$. The bar indicates $5 \mu \mathrm{m}$. All experiments were repeated at least three times and similar results were obtained

1k). By comparison, both $\mathrm{Ba}^{2+}$ and Spm were similarly effective in protecting chloroplasts against 16:1-induced rupture (Figure $1 \mathrm{~m}$ ) and cyt $f$ release (Figure 1n, 10).

Cyt $c$ has been found to be able to directly affect nuclei. ${ }^{16,17}$ As 16:1 affects in vivo nuclei (Figure 1a, b), we used isolated nuclei to examine whether $16: 1$ exerts its effect directly or via other intermediates. Nuclei incubated in cytosolic extract from untreated protoplasts and stained with DAPI had a bright blue florescence and a smooth appearance (Figure 1p). 16:1 had no direct effect on nuclei, indicating that the effects of $16: 1$ were mediated by other factor(s) (Figure 1p). Indeed, nuclei incubated in cytosolic extract from 16:1-treated protoplasts 
showed a reduction in fluorescence and nuclear volume (Figure 1p), suggesting that the extract contained the factor(s) affecting the nuclei. Similarly, stromal extract from untreated chloroplasts had no effect (Figure 1p), but extract from 16:1-treated chloroplasts did (Figure 1p), suggesting tht the factor affecting the nuclei could be found in the 16:1-treated chloroplasts. Turnip cyt $f$ obtained from Sigma ${ }^{\circledR}$, St. Louis, USA, produced effects similar to that caused by the extracts of 16:1-treated chloroplasts or protoplasts (Figure 1p). As cyt $f$ was found only in the supernatants of the 16:1-treated chloroplasts and protoplasts, the results taken together suggest that cyt $f$ was an intermediate responsible for the 16:1 effects.

In summary, this study demonstrates that cyt $f$ release induced by $16: 1$ is accompanied by cell death. Preventing cyt $f$ release by $\mathrm{Ba}^{2+}$ and Spm could at various degrees protect the protoplasts and chloroplasts from collapse. 16:1 does not directly caused damage of nuclei, but extracts from 16:1 treated protoplasts and chloroplasts do. As both of these extracts contain cyt $f$ that can directly cause damage to isolated nuclei, the results suggest that released cyt $f$ plays a role in cell death.

\section{Acknowledgements}

The antibodies to cyt $f$ used in this study were kindly provided by JE Thompson, University of Waterloo, Waterloo, ON, Canada. We thank MD
Smith, University of Massachusetts, Amherst, MA, USA for helpful discussions about this manuscript.

\section{JS Peters ${ }^{1}$ and C Chin ${ }^{*, 1}$}

${ }^{1}$ Department of Plant Biology and Pathology, Rutgers, The State University of New Jersey, New Brunswick, NJ, USA

* Corresponding author: C Chin, Department of Plant Biology and Pathology, Rutgers, The State University of New Jersey, New Brunswick, NJ 08901-8520, USA. Fax: 932-732-9441; E-mail: chin_c@ aesop.rutgers.edu

1. Green DR et al. (1998) Science 281: 1309-1312

2. Adrain C et al. (2001) Trends Biochem. Sci. 26: 390-397

3. Bratton SB et al. (2001) Trends Pharmacol. Sci. 22: 306-315

4. Woltering EJ et al. (2002) Plant Physiol. 130: 1764-1769

5. Jones A (2000) Trends Plant Sci. 5: 225-230

6. Tiwari BS et al. (2002) Plant Physiol. 128: 1271-1281

7. Thornberry NA et al. (1998) Science 281: 1312-1316

8. Barker PD et al. (1999) Structure 7: 281-290

9. Peters JS et al. (2003) Plant Physiol. Biochem. 41: 117-124

10. Gray J et al. (2002) Plant Physiol. 130: 1894-1907

11. Mach JM et al. (2001) Proc. Natl. Acad. Sci. USA 98: 771-776

12. Gavrieli $Y$ et al. (1992) J. Cell Biol. 119: 493-501

13. Joyard J et al. (1984) J. Biol. Chem. 258: 10000-10006

14. Ott M et al. (2002) Proc. Natl. Acad. Sci. USA 99: 1259-1263

15. Maeno E et al. (2000) Proc. Natl. Acad. Sci. USA 97: 9487-9492

16. Liu $X$ et al. (1996) Cell 86: 147-157

17. Zhao Y et al. (1999) FEBS Lett. 448: 197-200 